\title{
Bioscavengers for the protection of humans against organophosphate toxicity ${ }^{\text {is }}$
}

\author{
Bhupendra P. Doctor*, Ashima Saxena \\ Division of Biochemistry, Walter Reed Army Institute of Research, 503 Robert Grant Road, Silver Spring, MD 20910, USA
}

Available online 15 November 2005

\begin{abstract}
Current antidotes for organophosphorus compounds (OP) poisoning consist of a combination of pretreatment with carbamates (pyridostigmine bromide), to protect acetylcholinesterase (AChE) from irreversible inhibition by OP compounds, and post-exposure therapy with anti-cholinergic drugs (atropine sulfate) to counteract the effects of excess acetylcholine and oximes (e.g., 2-PAM chloride) to reactivate OP-inhibited AChE. These antidotes are effective in preventing lethality from OP poisoning, but they do not prevent post-exposure incapacitation, convulsions, seizures, performance decrements, or in many cases permanent brain damage. These symptoms are commonly observed in experimental animals and are likely to occur in humans.

The problems intrinsic to these antidotes stimulated attempts to develop a single protective drug, itself devoid of pharmacological effects, which would provide protection against the lethality of OP compounds and prevent post-exposure incapacitation. One approach is the use of enzymes such as cholinesterases (ChEs), $\beta$-esterases in general, as single pretreatment drugs to sequester highly toxic OP anti-ChEs before they reach their physiological targets. This approach turns the irreversible nature of the OP: ChE interaction from disadvantage to an advantage; instead of focusing on OP as an anti-ChE, one can use ChE as an anti-OP. Using this approach, it was shown that administration of fetal bovine serum AChE (FBSAChE) or equine serum butyrylcholinesterase (EqBChE) or human serum BChE (HuBChE) protected the animals from multiple $\mathrm{LD}_{50} \mathrm{~s}$ of a variety of highly toxic OPs without any toxic effects or performance decrements.

The bioscavengers that have been explored to date for the detoxification of OPs fall into three categories: (A) those that can catalytically hydrolyze OPs and thus render them non-toxic, such as OP hydrolase and OP anhydrase; (B) those that stoichiometrically bind to OPs, that is, $1 \mathrm{~mol}$ of enzyme neutralizes one or $2 \mathrm{~mol}$ of OP inactivating both, such as ChEs and related enzymes; and (C) and those generally termed as "pseudo catalytic", e.g., a combination of ChE and an oxime pre-treatment such that the catalytic activity of OP-inhibited ChE can rapidly and continuously be restored in the presence of an oxime. Since the biochemical mechanism underlying prophylaxis by exogenous esterases such as ChEs is established and tested in several animal species, including non-human primates, this concept should allow a reliable extrapolation of results from animal experiments to human application.

Having being extensively investigated by several groups, plasma derived HuBChE is judged to be the most suitable bioscavenger for its advancement for human use. The program is being developed at the present time for conducting a safety clinical trial in human volunteers. Several other candidate bioscavengers will follow; e.g., recombinant HuBChE expressed in the milk of transgenic goats, pseudo catalytic scavenger(s), e.g., a combination of ChE and oxime, and possibly PON 1 as a catalytic scavenger in the future. (C) 2005 Elsevier Ireland Ltd. All rights reserved.
\end{abstract}

Keywords: Butyrylcholinesterase; Bioscavengers; Organophosphorus compound toxicity; Efficacy

\footnotetext{
is The opinions or assertions contained herein are private views of the authors and are not to be construed as official or as reflecting the views of the US Army or the Department of Defense.

* Corresponding author. Tel.: +1 301319 9548; fax: +1 3013199150.

E-mail address: Bhupendra.Doctor@US.army.mil (B.P. Doctor).
} 


\section{Introduction}

Current antidotal regimens for organophosphorus compounds (OP) poisoning consist of a combination of pretreatment with a spontaneously reactivating acetylcholinesterase (AChE) inhibitor such as pyridostigmine bromide, and post-exposure therapy with anticholinergic drugs such as atropine sulfate and oximes such as 2PAM chloride [1]. Although these antidotal regimens are effective in preventing lethality in animals from OP poisoning, they do not prevent post-exposure incapacitation, convulsions, performance deficits or in many cases, permanent brain damage [2-4]. These problems stimulated the development of enzyme bioscavengers as a pretreatment to sequester highly toxic OPs before they reach their physiological targets and prevent the in vivo toxicity of OPs and post-exposure incapacitation [4]. Among the enzymes examined as potential scavengers of highly toxic OP nerve agents, significant advances have been made using cholinesterases (ChEs). Of the ChEs evaluated so far, human serum BChE (HuBChE) has several advantages as an exogenously administered prophylactic for human use [5]. First, it reacts rapidly with all highly toxic OPs, offering a broad range of protection for nerve agents including soman, sarin, tabun and VX. Second, it possesses a very long retention time in human circulation and is readily absorbed from sites of injection. Third, since the enzyme is from a human source, it should not produce any adverse immunological responses upon repeated administration to humans. A dose of $200 \mathrm{mg}$ of $\mathrm{HuBChE}$ is envisioned as a prophylactic treatment in humans that can protect from exposure of $2-5 \times \mathrm{LD}_{50}$ of nerve agents [5]. In addition, since $\mathrm{HuBChE}$ catalyzes the hydrolysis of cocaine and short-acting muscle relaxants succinylcholine and mivacurium, it could be an effective treatment for cocaine intoxication, as well as succinylcholine- and mivacurium-induced apnea [5].

This presentation is a review of the investigation of the pharmacokinetics, stability, safety, toxicity, and efficacy of purified $\mathrm{HuBChE}$ in mice, guinea pigs and non-human primates. These results provide convincing data that the $\mathrm{HuBChE}$ is a safe and effective bioscavenger and therefore should be assessed for inclusion into the protective regimen against OP nerve agents.

\section{Results and discussion}

\subsection{Isolation, purification and characterization of $\mathrm{HuBChE}$}

The enzyme was isolated from outdated human plasma or from freshly produced Cohn fraction IV-4, using a variety of analytical methods. The yield of purified enzyme from various sources varies from $35-70 \%$. It is a tetrameric glycoprotein $(340 \mathrm{kDa})$ made up of four identical subunits. Each subunit consists of 575 amino acids and contains three disulfide bridges. Each subunit contains nine $\mathrm{N}$-linked glycosylation sites and one catalytic site. The active site serine is located at position 198 from the N-terminal. The partial structures of the carbohydrates present at each site are described elsewhere in these proceedings (see Garcia et. al., these proceedings). The purity of the enzyme can be followed by specific activity, SDS-PAGE, and HPLC. The specific activity of the enzyme is reported to be $700-750 \mathrm{U} / \mathrm{mg}$ when measured in $50 \mathrm{mM}$ sodium phosphate buffer at $\mathrm{pH}$ 8.0 , at $25^{\circ} \mathrm{C}$, using $1 \mathrm{mM}$ butyrylthiocholine as the substrate [6]. Active-site titration of purified enzyme with DEPQ revealed that $1 \mathrm{mg}$ of enzyme contains $11 \mathrm{nmol}$ of active-sites. Each nanomole of enzyme contains 64-68 U of activity. The $A_{280}$ of $1 \mathrm{mg} / \mathrm{ml}$ solution varies from $1.8-1.92[6,7]$. The enzyme migrates as a single band on a reducing SDS-PAGE, with a subunit molecular weight of $85 \mathrm{kDa}$. The size of the band is reduced to $65 \mathrm{kDa}$ upon treatment with $\mathrm{N}$-glycosidase F. The intact protein migrates as a single band on native polyacrylamide gel electrophoresis, which can be stained for enzyme activity. The intact protein also migrates as a single peak on sucrose density gradients, with a sedimentation coefficient $\left(s_{20, w}\right)$ of 12.44 , corresponding to the tetrameric form of the enzyme [6]. Similarly, the enzyme migrates as a single peak on a gel permeation column. The isoelectric point of the enzyme was reported to be at $\mathrm{pH}$ 3.99 [7].

\subsection{In vitro and in vivo stability of $H u B C h E$}

The stability of the enzyme was determined at various temperatures. Aliquots of enzyme were stored in lyophilized $(1 \mathrm{mg})$ or liquid form $(10 \mathrm{mg} / \mathrm{ml}$ in $50 \mathrm{mM}$ sodium phosphate, $\mathrm{pH} 8.0+10 \%$ glycerol $+1 \mathrm{mM}$ EDTA) at $4,25,37$, or $45^{\circ} \mathrm{C}$. The enzyme was stable when stored in lyophilized form at $4,25,37$, or $45^{\circ} \mathrm{C}$ and in liquid form at 4 and $25^{\circ} \mathrm{C}$. The study was extended for over 2 years and the enzyme was found to be stable in lyophilized form at 4 and $25^{\circ} \mathrm{C}$.

The in vivo circulatory stability of the enzyme was determined by measuring the pharmacokinetic profile of the enzyme (stored at $-20^{\circ} \mathrm{C}$ for various time periods) following i.m. administration into mice. The pharmacokinetic properties of the enzyme were not affected upon storage at $-20^{\circ} \mathrm{C}$ to date (25 months). These results are described in details elsewhere in this proceeding (see Saxena et al., this volume). 


\subsection{Pharmacokinetics and bioavailability}

The following studies describe the pharmacokinetics and bioavailability of HuBChE in rodents and nonhuman primates.

\subsubsection{Mice}

The maximum dose of $\sim 90 \mathrm{mg} / \mathrm{kg}$ ( $3 \mathrm{mg} / \mathrm{mouse}$ ) was administered into CD-1 mice by a single i.p. or i.m. injection. Blood samples were taken at 12 time points for up to 14 days post-HuBChE injection for the monitoring of blood BChE activity using the Ellman assay. The following pharmacokinetic parameters were determined from the time course curve of blood BChE concentration: mean retention time (MRT), maximal concentration $\left(C_{\max }\right)$, time to reach the maximal concentration $\left(T_{\max }\right)$, elimination half-life $\left(T_{1 / 2}\right)$, and area under the plasma concentration time curve extrapolated to infinity (AUC). Purified $\mathrm{HuBChE}$ exhibited circulatory stability profiles similar to those observed previously for enzyme purified from human plasma in rats and mice [8], guinea pigs [9] and rhesus monkeys [10]. Mice that were administered 70-2100 U of HuBChE by i.p. injection showed a rapid increase in BChE activity, which reached peak levels at $10 \mathrm{~h}$. On the other hand, when the same doses of enzyme were delivered by i.m. injections, peak levels of activity were attained at $\sim 24 \mathrm{~h}$. Regardless of the dose and route of administration, the enzyme displayed an MRT of $45-53 \mathrm{~h}$. These results are summarized in Table 1 and described in detail in these proceedings (see Saxena et. al. this volume).

\subsubsection{Guinea pigs}

(a) Two doses of HuBChE (19.9 and $32.5 \mathrm{mg} / \mathrm{kg}$ ) were injected i.m. in guinea pigs to determine its pharmacokinetics in blood. Mean whole blood $\mathrm{HuBChE}$ time-concentration data were fit to stan- dard pharmacokinetic models. The pharmacokinetics of HuBChE in guinea pigs followed a onecompartment model with a single elimination phase (for details, see Lenz et al., this volume).

(b) Sixty milligrams per kilograms of HuBChE was administered to guinea pigs by a single i.p. or i.m. injection. Blood samples were taken at 18 time points following HuBChE injection for the measurement of blood BChE activity. The pharmacokinetic parameters of $\mathrm{HuBChE}$ administrated by two different routes are summarized in Table 1 (for details see Sun et al., this volume).

\subsubsection{Monkeys}

(a) Two doses of $\mathrm{HuBChE}$ (5.25 and $8.75 \mathrm{mg} / \mathrm{kg}$ ) were injected i.m. in cynomolgus monkeys to determine its pharmacokinetics in blood. The pharmacokinetics of $\mathrm{HuBChE}$ in cynomolgus monkeys also fit a one-compartment model with a single elimination phase. The pharmacokinetic parameters are summarized in Table 1.

(b) HuBChE $(30 \mathrm{mg} / \mathrm{kg})$ was injected i.v. in four rhesus monkeys. The zero time baseline mean was $4.3 \mathrm{U} / \mathrm{ml}$. At 5-min post-injection, the mean level of BChE in blood was $225.6 \mathrm{U} / \mathrm{ml}$. The mean residence time was $72 \mathrm{~h}$. By 2 weeks post-injection, the circulating level of BChE $(4.4 \mathrm{U} / \mathrm{ml})$ approximated baseline levels. The pharmacokinetic parameters are summarized in Table 1.

\subsection{Safety and toxicity}

Blood samples from mice, guinea pigs and rhesus monkeys, obtained prior to administration of $\mathrm{HuBChE}$ and 14 days post-injection, were analyzed for serum chemistry (including glucose, urea nitrogen, creatinin, $\mathrm{Na}, \mathrm{K}, \mathrm{Cl}, \mathrm{CO}_{2}, \mathrm{Ca}, \mathrm{PHOS}$, cholesterol, triglycerides,

Table 1

Pharmacokinetic parameters of $\mathrm{HuBChE}$ in monkeys, guinea pigs, and mice

\begin{tabular}{|c|c|c|c|c|c|c|c|}
\hline \multirow[t]{2}{*}{ Parameters } & \multicolumn{2}{|l|}{ Monkeys } & \multicolumn{3}{|l|}{ Guinea pigs } & \multicolumn{2}{|l|}{ Mice } \\
\hline & i.v. ${ }^{a}$ & i.m. ${ }^{\text {b }}$ & i.p..$^{c}$ & i.m. ${ }^{c}$ & i.m. ${ }^{\text {b }}$ & i.p. ${ }^{\text {d }}$ & i.m. ${ }^{d}$ \\
\hline MRT (h) & $72 \pm 7$ & NR & $109 \pm 6$ & $110 \pm 4$ & NR & $42 \pm 3$ & $51 \pm 2$ \\
\hline$T_{1 / 2}(\mathrm{~h})$ & $36 \pm 7$ & $74 \pm 4$ & $63 \pm 7$ & $62 \pm 4$ & $76 \pm 10$ & $11 \pm 1$ & $16 \pm 1$ \\
\hline$T_{\max }(\mathrm{h})$ & 0 & $10 \pm 1$ & $30 \pm 2$ & $27 \pm 2$ & $27 \pm 2$ & $14 \pm 5$ & $24 \pm 0$ \\
\hline$C_{\max }(\mathrm{U} / \mathrm{ml})$ & $222 \pm 24$ & $33 \pm 6$ & $293 \pm 12$ & $258 \pm 12$ & $21 \pm 6$ & $364 \pm 51$ & $225 \pm 23$ \\
\hline AUC (U h/ml) & $16538 \pm 2155$ & $3822 \pm 133$ & $39163 \pm 1714$ & $35196 \pm 2084$ & $26880 \pm 2310$ & $20279 \pm 1428$ & $16162 \pm 1258$ \\
\hline
\end{tabular}

\footnotetext{
a Myers et al. [11].

b Lenz et al., this volume.

c Sun et al., this volume.

d Saxena et al., this volume.
} 
Table 2

Efficacy of HuBChE in guinea pigs and monkeys

\begin{tabular}{lllll}
\hline Protection & Species & Toxin & Dose $\left(\right.$ LD $\left._{50}\right)$ & Recovery \\
\hline HuBChE & Guinea pigs $(n=9)$ & VX & 5.0 & Immediate \\
HuBChE & Guinea pigs $(n=10)$ & GD & 5.5 & Immediate \\
HuBChE & Rhesus monkey & GD & 3.3 & Immediate \\
HuBChE & Cynomolgus monkey $(n=6)$ & GD & 5.5 & 4 of 6 \\
\hline
\end{tabular}

${ }^{a}$ Raveh et al. [10].

total protein, albumin AST, ALT, LDH, CK, ALKP, GGT, and total bilirubin) and hematology (including WBC, RBC, HGB, HCT, MCV, MCH, MCHC, RDW, PLT, and MPV) parameters. Mice and guinea pigs were euthanized at 14 days post-injection of $\mathrm{HuBChE}$ and complete gross and histopathology examinations were conducted. No toxic effects were suggested from the observed parameters.

\subsubsection{Efficacy studies}

The following studies describe the efficacy studies conducted with $\mathrm{HuBChE}$ in guinea pigs and non-human primates.

\subsubsection{Guinea pigs}

Guinea pigs $(n=10)$ were administered HuBChE by i.m. injection in sufficient quantities to neutralize an $8 \times \mathrm{LD}_{50}$ challenge of GD $\left(1 \times \mathrm{LD}_{50}=30 \mu \mathrm{g} / \mathrm{kg}\right.$ s.c. $)$ or VX $\left(1 \times \mathrm{LD}_{50}=9 \mu \mathrm{g} / \mathrm{kg}\right.$ s.c. $)$ calculated on the basis of 1:1 stoichiometry between the enzyme and OP. At $19( \pm 1.0) \mathrm{h}$ post-enzyme administration, a blood sample was taken and the whole blood $\mathrm{BChE}$ concentration was determined. Based on the data, the number of moles of $\mathrm{BChE}$ in circulation of guinea pigs was determined and the animals were challenged with either GD or VX. The challenge design allowed for multiple exposures of experimental animals with lethal amounts of GD or VX until a total of $5.5 \times \mathrm{LD}_{50}$ dose was administered. In case of GD challenge, all animals $(n=10)$ survived with no observable signs (Table 2). At necropsy, either 7 days $(n=5)$ or 14 days $(n=5)$ post-GD challenge, all organs appeared normal and no abnormal histopathology was observed in hematoxylin and eosin stained tissues. Similarly, when animals were challenged with VX $(n=9)$ under a similar paradigm, all animals survived the challenge doses of $\mathrm{VX}$, for a total of $5.0 \times \mathrm{LD}_{50}$ with no observable sign of intoxication (Table 2 ; for details see Lenz et al., this volume).

\subsubsection{Cynomolgus monkeys}

Six cynomolgus monkeys received $75 \mathrm{mg}$ of $\mathrm{HuBChE}$ followed by sequential doses $\left(1.5,2.0,2.0 \times \mathrm{LD}_{50}\right)$ of soman $10 \mathrm{~h}$ later for a total challenge of $5.5 \times \mathrm{LD}_{50}$ of soman. One animal died after the 3rd dose of GD; another was impaired and euthanized after $48 \mathrm{~h}$. Four animals that did not show any signs of soman intoxication (Table 2) were transferred to the Walter Reed Army Institute of Research for the evaluation of long-term health effects. Each month, blood was drawn from these monkeys for the analysis of serum chemistry and hematology parameters, blood $\mathrm{BChE}$ levels and anti-BChE production. No toxic effects or any organ malfunctions have been observed so far.

\section{Conclusions}

The present investigations were designed to fulfill the US Food and Drug Administration regulatory requirements and the clinical compliance for testing and evaluating plasma-derived $\mathrm{HuBChE}$ as a pretreatment for the toxicity of OPs in humans. A procedure to acquire large quantities of purified $\mathrm{HuBChE}$ from Cohn fraction IV-4 was developed. This process yielded 5-8 g of enzyme from approximately 80 to $100 \mathrm{~kg}$ of Cohn fraction IV-4 paste. The process was essentially akin to cGMP conditions. Analysis of the product yielded results that agreed with previously obtained and reported properties of this enzyme. In addition, this product was stable for more than 2 years when stored in lyophilized form at $-20^{\circ} \mathrm{C}$. Also, it retained its in vivo bioavailability for over 2 years. The average MRT of the enzyme appeared to be $\sim 50 \mathrm{~h}$ in mice. The MRT in guinea pigs was approximately $100-110 \mathrm{~h}$ whereas the MRT in nonhuman primates was approximately $70 \mathrm{~h}$. Extrapolation of these results and the instances where HuBChE was used as a treatment for pesticide poisoning in human patients suggest that the MRT in humans may approach to $12-15$ days.

With regard to the efficacy of $\mathrm{HuBChE}$ in guinea pigs and non-human primates, pretreatment of animals with $\mathrm{HuBChE}$ and subsequent s.c. challenge with soman or VX resulted in a stoichiometric sequestration of OPs. Similar results were previously observed in mice $[8,12]$, rats [13], guinea pigs [9], and rhesus monkeys [10]. 
Although $1 \mathrm{~mol}$ of HuBChE will sequester $1 \mathrm{~mol}$ of OP, the rate of sequestration of different OPs by $\mathrm{HuBChE}$ does not appear to be the same. In guinea pigs, it takes longer for VX to be sequestered by BChE than soman. Extrapolation of data obtained from prophylaxis experiments with $\mathrm{HuBChE}$ in four species suggests that a dose of $200 \mathrm{mg}$ of $\mathrm{HuBChE}$ as a prophylactic treatment can protect humans from an exposure of up to $2 \times \mathrm{LD}_{50}$ of nerve agents [5].

Most efficacy studies conducted to date utilized i.v. or s.c. challenge of OPs. A study in which guinea pigs were administered soman by inhalation challenge following pretreatment with $\mathrm{HuBChE}$ (i.v. or i.m.) showed that only $26-30 \%$ of the enzyme was neutralized (14). Almost one quarter of the soman was exhaled and one half was neutralized elsewhere in the upper respiratory tract. It is most likely that the human exposure of OPs will be experienced by inhalation (>90\%). Therefore, more efficacy studies need to be conducted using OP challenge by inhalation before one can establish the dose of enzyme that will be required to protect humans from an exposure of $5 \times \mathrm{LD}_{50}$ of nerve agents.

A critical prerequisite for any potential bioscavenger is a prolonged circulatory residence time and the absence of anti-enzyme antibodies following repeated injections of the enzyme. Previously, it was demonstrated that multiple injections of equine BChE into rabbits, rats, or rhesus monkeys resulted in an MRT spanning several days, and the induction of anti-enzyme antibodies [14-17]. In these experiments, blood enzyme activity appeared to correlate negatively with anti-BChE IgG levels. On the other hand, administration of purified macaque $\mathrm{BChE}$ into macaques of the same species resulted in a much longer MRT $=225 \pm 19 \mathrm{~h}$ compared to that reported for heterologous HuBChE $(33.7 \pm 2.9 \mathrm{~h})$ was observed. A smaller second injection of macaque $\mathrm{BChE}$ given 4 weeks later attained predicted peak plasma levels of enzyme activity, although the four macaques showed wide variation in the MRT $=54-357 \mathrm{~h}$. No antibody response was detected in macaques following either injection of enzyme [18]. These results support the development of human $\mathrm{BChE}$ as a bioscavenger for OP toxicity in humans.

\section{References}

[1] A.P. Gray, Design and structure-activity relationships of antidotes to organophosphorus anticholinesterase agents, Drug Metab. Rev. 15 (1984) 557-589.
[2] P. Dirnhuber, M.C. French, D.M. Green, I. Leadbeater, J.A. Stratton, The protection of primates against soman poisoning by pretreatment with pyridostigmine, J. Pharm. Pharmacol. 31 (1979) 295-299.

[3] C.G. McLeod, Pathology of nerve agents: perspectives on medical management, Fundam. Appl. Toxicol. 5 (1985) S10-S16.

[4] M.A. Dunn, F.R. Sidell, Progress in medical defense against nerve agents, J. Am. Med. Assoc. 262 (1989) 649-652.

[5] Y. Ashani, Prospective of human butyrylcholinesterase as a detoxifying antidote and potential regulator of controlled-release drugs, Drug Dev. Res. 50 (2000) 298-308.

[6] O. Lockridge, H.W. Eckerson, B.N. La Du, Interchain disulfide bonds and subunit organization in human serum cholinesterase, J. Biol. Chem. 254 (1979) 8324-8330.

[7] O. Lockridge, B.N. La Du, Comparison of atypical and usual human serum cholinesterase. Purification, number of active sites, substrate affinity, and turnover number, J. Biol. Chem. 253 (1978) 361-366.

[8] L. Raveh, J. Grunwald, D. Marcus, Y. Papier, E. Cohen, Y. Ashani, Human butyrylcholinesterase as a general prophylactic antidote for nerve agent toxicity, Biochem. Pharmacol. 45 (1993) 2465-2474.

[9] N. Allon, L. Raveh, E. Gilat, E. Cohen, J. Grunwald, Y. Ashani, Prophylaxis against soman inhalation toxicity in guinea pigs by pretreatment alone with human serum butyrylcholinesterase, Toxicol. Sci. 43 (1998) 121-128.

[10] L. Raveh, E. Grauer, J. Grunwald, E. Cohen, Y. Ashani, The stoichiometry of protection against soman and VX toxicity in monkeys pretreated with human butyrylcholinesterase, Toxicol. Pharmacol. 145 (1997) 43-53.

[11] T.M. Myers, W. Sun, W.R. Bansal, M.G. Clark, A. Saxena, B.P. Doctor, Safety evaluation of human serum butyrylcholinesterase in Rhesus monkeys, Proc. Jt. Serv. Sci. Conf. Chem. Biol. Def. Res. 1 (2003) 1-8.

[12] Y. Ashani, S. Shapira, D. Levy, A.D. Wolfe, B.P. Doctor, L. Raveh, Butyrylcholinesterase and acetylcholinesterase prophylaxis against soman poisoning in mice, Biochem. Pharmacol. 41 (1991) 37-41.

[13] R. Brandeis, L. Raveh, J. Grunwald, E. Cohen, Y. Ashani, Prevention of soman-induced cognitive deficits by pretreatment with human butyrylcholinesterase in rats, Pharmacol. Biochem. Behav. 46 (1993) 889-896.

[14] R.F. Genovese, X-C. Lu, M.K. Gentry, R. Larrison, B.P. Doctor, Evaluation of purified horse serum butyrylcholinesterase in rats, Proc. Med. Def. Biosci. Rev. 3 (1993) 1035-1042.

[15] M.K. Gentry, E.S. Nuwayser, B.P. Doctor, Effects of repeated administration of butyrylcholinresterase on antibody induction in rabbits, Proc. Med. Def. Biosci. Rev. 3 (1993) 1051-1056.

[16] M.K. Gentry, E.S. Nuwayser, B.P. Doctor, Immunological effect of repeated administration of cholinesterases in rabbits, Proc. Med. Def. Biosci. Rev. 1 (1996) 183-191.

[17] S.M. Matzke, J.L. Oubre, G.R. Caranto, M.K. Gentry, G. Galbicka, Behavioral and immunological effects of exogenous butyrylcholinesterase in rhesus monkeys, Pharmacol. Biochem. Behav. 62 (1999) 523-530.

[18] Y. Rosenberg, C. Luo, Y. Ashani, B.P. Doctor, R. Fischer, G. Wolfe, A. Saxena, Pharmacokinetics and immunologic consequences of exposing macaques to purified homologous butyrylcholinesterase, Life Sci. 72 (2002) 125-134. 